\title{
AI NOSTRI LETTORI
}

Gli Autori e la Casa Editrice dell'Anatomia Patologica Speciale di E. KaUfmann si propongono di pubblicare, accanto al completamento della II $^{\text {a }}$ e I $^{\text {a }}$ edizione

\section{Supplementi di aggiornamento delle parti finora edite}

che rechino i risultati delle ricerche recenti, apparse dopo la loro pubblicazione. $\grave{E}$ previsto di dare maggiore sviluppo alle conoscenze di microscopia elettronica $e$ di istochimica, ma anche di fornire brevi sintesi di particolari problemi, che abbiano avuto rilievo nella letteratura degli ultimi anni. La loro elaborazione viene condotta in parte dagli Autori delle parti finora apparse, in parte da Autori che appositamente hanno offerto la loro opera a questo fine. Nell'ambito del

\section{Primo volume di supplemento}

il prof. W. DoERr, Heidelberg, completerà la sua sintesi, universalmente apprezzata, delle malformazioni del cuore e dei grossi vasi. Il prof. ScHumMELFEDER di Colonia, prematuramente scomparso, aveva assunto il compito di elaborare problemi di istochimica e di microscopia in fluorescenza del cuore e dei vasi sanguigni. Il dr. CAESAR di Tubingen tratterà la microscopia elettronica del cuore. Il prof. Schoenmackers di Aquisgrana ha accettato di elaborare il problema delle arterie coronarie e della irrorazione sanguigna del muscolo cardiaco, il prof. SANDRITTER di Giessen, la morfologia della trombosi. Altre parti dovrebbero riguardare le necrosi chimiche del muscolo cardiaco, la miocardite, e le malattie delle vene, in particolare la flebite.

Speriamo con questi contributi di poter essere ulteriormente utili ai nostri lettori sempre nel senso di trovare nel KaUFmanN il consigliere, che renda loro familiari le più recenti conoscenze scientifiche nel campo della patologia morfologica. 
